\title{
Liquid water uptake in unconfined Callovo Oxfordian clay-rock studied with neutron and x-ray imaging
}

\author{
Eleni Stavropoulou ${ }^{1,2, \dagger}$, Edward Andò ${ }^{1}$, Alessandro Tengattini ${ }^{1,3}$, Matthieu \\ Briffaut $^{1}$, Frédéric Dufour ${ }^{1}$, Duncan Atkins ${ }^{3}$, and Gilles Armand ${ }^{2}$ \\ ${ }^{1}$ Univ. Grenoble Alpes, CNRS, Grenoble INP*, 3SR, F-38000, Grenoble, France \\ ${ }^{2}$ Agence Nationale pour la gestion des Déchets Radioactifs (Andra), \\ Châtenay-Malabry, France \\ ${ }^{3}$ Institute Laue-Langevin, 71 avenue des Martyrs - CS 20156, 38042 Cedex 9 \\ Grenoble, France \\ †eleni.stavropoulou@3sr-grenoble.fr
}

\begin{abstract}
The Callovo Oxfordian clay-rock (COx) is studied in France for the disposal of radioactive waste, because of its extremely low permeability. This host rock is governed by a hydromechanical coupling of high complexity. This paper presents an experimental study into the mechanisms of water uptake in small, unconfined, prismatic specimens of COx, motivated by the comprehension of cracking observed during concrete/COx interface sample preparation. Water uptake is monitored using both x-ray tomography and neutron radiography, the combination of these imaging techniques allowing material deformation and water arrival to be quantified respectively. Given the speed of water entry and crack propagation, relatively fast imaging is required: 5 minute x-ray tomographies and ten-second neutron radiographies are used. In this study, pairs of similar COx samples from the same core are tested separately with each imaging technique. Two different orientations with respect to the core are also investigated. Analysis of the resulting images yields with micro- and macro-scale insights into hydro-mechanical mechanisms to be obtained. This allows the cracking to be interpreted as a rapid breakdown in capillary suction (supposed large both to drying and rebound from in-situ stress state) due to water arrival, which in turn causes a loss of effective stress, allowing cracks to propagate with ease, which in turn deliver water further into the material.
\end{abstract}

\section{Introduction}

The management of radioactive waste is an important environmental issue, in particular in the countries where nuclear power is generated. In Eastern France, the French National Radioactive Waste Management Agency (Andra) is studying the behaviour of a deep geological repository in Callovo Oxfordian clay-rock (COx). The permeability of the Callovo Oxfordian stratum is extremely low, it is however governed by complex thermo-hydro-mechanical behaviour, which is continuously under investigation with both laboratory and in-field experiments ([1]-[4]).

During the operation phase, tunnels will be excavated and ventilated, until their final closure. In specific sections, swelling clay like bentonite, will be emplaced in between two concrete plugs in order to seal the tunnel and apply pressure on the rock wall, in sections where the concrete lining will be removed $([5,6])$. The study of the mechanical behaviour of the concrete/COx interface in the lab using among others the newly-developed SInC box [7], requires representative and reproducible concrete/COx samples to be created under controlled conditions.

During the preparation of these samples when fresh concrete has been poured directly onto the unconfined COx, macro-cracks have been detected before starting mechanical experiments. Many authors have studied the effect of drying and wetting cycles on different clay-rocks; on Callovo Oxfordian clay-rock samples with saline solution $([8,9,10])$ and in situ on Tournemire

\footnotetext{
*Institute of Engineering, Univ Grenoble Alpes
} 
clay-rock ([11, 12]) and on Callovo Oxfordian clay-rock ([13]). Guillon et al [10] have performed static atmosphere drying tests which highlighted the hydro mechanical coupling and mechanical anisotropy of the samples. Both wetting and drying paths were explored, allowing the potential hysteretic behaviour of the samples. During imbibition, the swelling of the sample is mainly achieved by the interaction of water with swelling clay sheets (illite/smectite interstratified, [14]). The opening of some cracks can also be responsible for the dilation of the sample. At a smaller scale, Wang et al [15] also studied the evolution of the COx clay-rock under hydric cycles using techniques based on the combination of environmental scanning electron microscope (ESEM) and digital image correlation (DIC) [9], in order to quantify local strain field. The observation is carried out on zones of several hundred micrometers, so that the evolution of such material under hydric loadings can be studied at the scale of inclusion-matrix composite. A heterogeneous strain field is evidenced at the microscale during wetting. Nonlinear deformation is observed at high relative humidity $(\mathrm{RH})$ which is related not only to damage, but also to the nonlinear swelling of the clay mineral itself, controlled by different local mechanisms depending on relative humidity. This sensitivity to variations in water content leads to enhanced variations of mechanical parameters in response to water saturation.

In the case of poured concrete/clay-rock interface, the cause of these macro-cracks must be further investigated in order to understand whether they reflect site conditions, and furthermore since their presence may affect the representativeness of the measured mechanical response in the lab. In the lab (and presumably on site) the exposed, drying face of the clay-rock is rapidly wetted by the free water of the fresh concrete. To gain some insight into the causes of this phenomenon, a number of in-situ wetting experiments have been carried out with high-speed lab x-ray tomography (in Laboratoire 3SR) and neutron radiography (at the ILL instrument D50-tomo or "NeXT", an instrument that has recently come online in Grenoble [16]).

\section{$2 \quad$ In-situ imaging techniques}

Full-field imaging is a very precious tool in experimental mechanics especially for the observation and study of inhomogeneous phenomena. The use of penetrating radiation allows the inside of studied specimens to be revealed, which is a great improvement over photography for the representativeness of the measured field. The attenuation of penetrating radiation beam (of a given energy) as it travels through matter can often be modelled using the Beer-Lambert law, here given for a specimen made of one material:

$$
I=I_{0} e^{-\mu_{m} \rho x}
$$

where $I$ is the resulting beam intensity after interaction with the material, $I_{0}$ is the reference beam intensity without interaction (no sample), $\mu_{m}$ is the mass attenuation coefficient ( $\mu_{m}=\mu / \rho$, where $\mu$ is the linear attenuation coefficient which is a material property), $\rho$ is the density of the material and $x$ is the linear distance that the beam travels inside the specimen.

Furthermore, the use of penetrating radiation enables the use of tomographic techniques, which give access to the attenuation field - a 3D "image" of $\mu$.

In this work we present full-field measurements of water imbibition into a clay-rock observed with two highly complementary technqiues using different types of penetrating radiation - x-ray tomography and neutron radiography - both of which will be briefly described below.

\subsection{X-ray tomography}

X-rays are massless photons with high-energy that interact with the electron cloud surrounding each atom's nucleus. Therefore the probability of an x-ray photon being attenuated is proportional to the atomic number $\mathrm{Z}$, which itself is roughly proportional to the density of the material.

Tomography consists in sampling the unknown attenuation field within a specimen from a large number of different orientations, which allows an accurate reconstruction of the desired attenuation field using back-projection.

Tomography was developed with x-ray radiation, and x-ray tomography remains the most developed form of tomography with high-performance laboratory scanners and dedicated synchrotron installations. 
Within mechanics in general, and geomechanics in particular, it has become a de-facto standard for time-resolved 3D analysis. For example, [17] presents the in-situ deformation mechanisms in Callovo-Oxfordian clay-rock in triaxial compression using x-ray tomography with important results regarding strain localisation. The measurement of strain in this material is possible due to the natural inhomogeneities within the rock, that present a convenient pattern tracking with image correlation. The observed patterns reveal that the different components that make up the rock must have significantly different densities.

X-ray tomography (using a lab-based micro-focus scanner) will be used in this work to acquire a number of 3D x-ray attenuation fields during the imbibition process, allowing the deformation of the rock to be measured.

However, due to the relatively low density of water, this technique will have difficulty measuring the invasion of water into the sample.

\subsection{Neutron radiography}

Neutrons are neutral massive particles that interact directly with the nucleus of atoms. Neutrons generally have a much higher probability of interaction with light atoms like hydrogen, even though attenuation can be radically different between isotopes of the same atom (for example deuterium has a much lower attenuation than hydrogen).

Neutrons principally interact with nuclei by being scattered or absorbed; when a neutron is scattered its speed and direction change, whereas when it is absorbed a wide range of radiations can be emitted or fission can be induced in some specific elements. The nucleus may rearrange its internal structure and release one or more gamma rays, charged particles may also be emitted. The sensitivity of neutrons to hydrogen make it an ideal complement to x-rays, significantly facilitating the detection of water throughout the imbibition process. It is important to note that in the interaction of neutrons with water, the principal mechanism is elastic scattering.

To obtain low-noise radiographies at the pertinent resolution for this phenomenon, neutron imaging is slow (even compared to a lab-based x-ray scanner), and so it has been elected to perform neutron radiography for this study, since the time required for neutron tomography was estimated to be too long.

Since a 3D field of neutron attenuation will not be reconstructed, great care has been taken to fully characterise the interaction of neutrons with water in the experimental conditions used for this test. Figure 1 shows a key step in this procedure where a hollow aluminium wedge has been scanned with neutrons before and after filling with $\mathrm{H}_{2} \mathrm{O}$, in order to fit the attenuation coefficient $\mu_{m}$ of neutrons to water, net of the whole imaging setup. The left of the Figure presents a schematic showing the setup. It is important to note that due to scattering, in neutron imaging the sample is kept as close to the detector as possible. The middle of the Figure shows the recorded radiographies of $I / I_{0}$ before and after filling the wedge with water. The change in attenuation of the beam between these two images is then extracted along the profile shown in violet in Figure 1 middle-bottom. Together with the knowledge of the geometry of the wedge, this allows the relationship between the thickness of water in the direction of the beam ( $x$ in Beer-Lambert) and its attenuation $\left(I / I_{0}\right)$, as shown in violet in Figure 1 right-bottom. This experimental data is fitted with the Beer-Lambert law (equation 1 ) with $\mu$ being the only free parameter. The fit is made in the range $[0,2.3] \mathrm{mm}$, that is to say until half of the "mean-free-path length" $(\lambda)$ of neutrons with wavelength of $1.8 \AA$ in $\mathrm{H}_{2} \mathrm{O}$ (close to the peak of $3 \AA$ in the beam used), as given in [18]; the fit gives $\mu=0.310$.

It is immediately apparent that for more than $3 \mathrm{~mm}$ of $\mathrm{H}_{2} \mathrm{O}$ there is a significant deviation between the fitted Beer-Lambert absorption and the measured attenuation data, this is likely due to scattering of the neutron beam. For short path lengths in the water (well below the mean-pathlength of $4.6 \mathrm{~mm}$ ), the attenuation of the beam is low, therefore there is a significant amount of transmission $\left(I / I_{0}\right)$. The non-transmitted beam is scattered and detected elsewhere, but since this is a small amount, the relationship between distance in water and attenuation is modelled well by Beer-Lambert. For longer path lengths, for example $10 \mathrm{~mm}$, a $5 \%$ of the beam is expected to be transmitted. This implies that almost $95 \%$ of the beam will be scattered, and the scattered beam itself will be scattered again in a phenomenon known as multiple scattering. Some of this diffused beam will be detected at the position of the transmitted beam, thus increasing the measured flux to 19\%. The effect of scattering is clearly visible in Figure 1 right-top, where the measured intensity in red green and blue zones increases when water is added. 

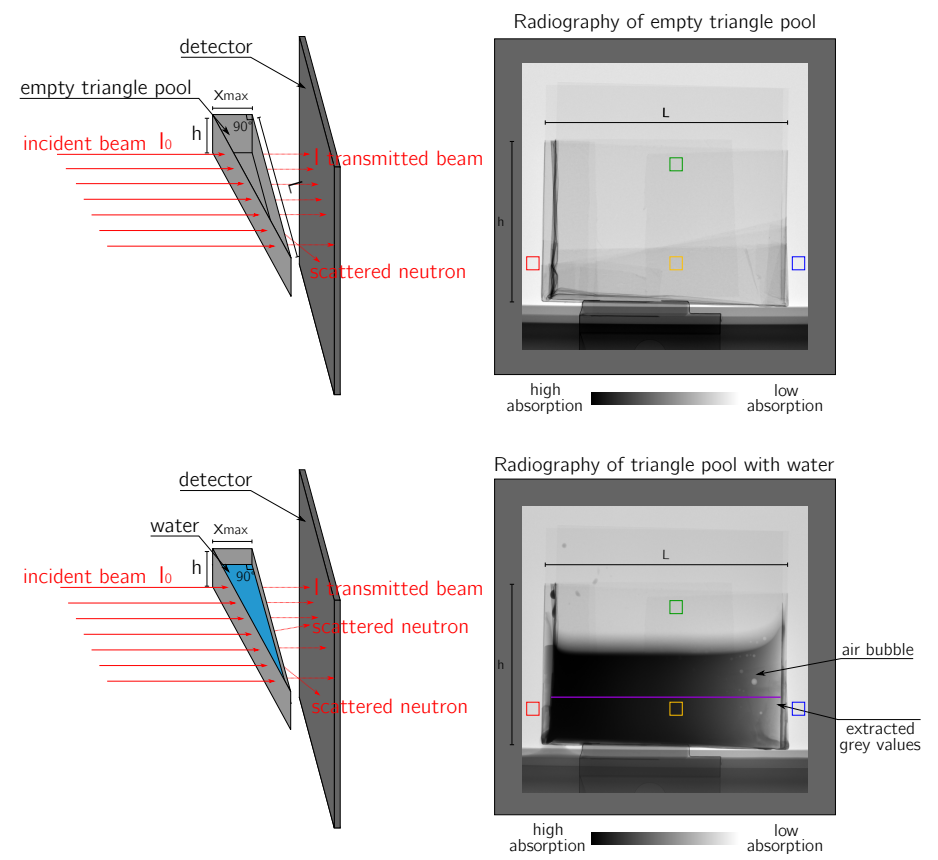
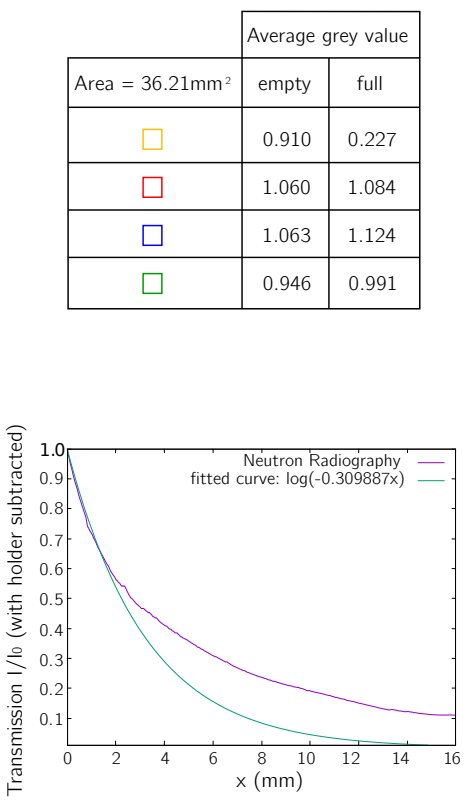

Figure 1: Calibration of the beam absorption in $\mathrm{H}_{2} \mathrm{O}$ with neutron path length, on the left, experimental schematic, in the middle experimental data, top-right grey value in some zones of interest, bottom-right the fit of the Beer-Lambert law to the acquired data

We therefore have a good degree of confidence in the Beer-Lambert law for up to $3 \mathrm{~mm}$ of water in the direction of the beam.

\section{Experimental Campaign}

During the preparation of concrete/COx interface specimens for mechanical testing, fresh concrete is poured directly onto the free surface of an unconfined cylinder of $\mathrm{COx}(78 \mathrm{~mm}$ in diameter, $50 \mathrm{~mm}$ height), causing cracking within one day.

This phenomenon was studied in a preliminary study using time-resolved x-ray tomography as shown in Figure 2 a); cracks visible after $1 \mathrm{~h}$ are highlighted in red in the vertical slices through the x-ray tomography volumes middle and bottom. The free-water in the concrete was suspected as the root cause of the cracking in the COx. In order to confirm the availability of free water as the main mechanism for cracking a second experiment (Figure $2 \mathrm{~b}$ ) was carried out, where the concrete was replaced by tap water provided to the top part of a COx specimen of the same dimensions as a). Again, cracks after one hour are highlighted in red, visually confirming the repeatability of the phenomenon. In both experiments cracking is clearly visible, and continues to an impressive degree after an hour, however in both cases the entry of water into the specimen is not visible, weakening the conclusions and hampering further interpretation.

Neutron imaging was selected as an ideal complement to x-ray tomography for the detection of water entry. Given the limitations of neutron radiography discussed above, prismatic specimens with one small dimension are clearly convenient.

Given the change of geometry of the specimen, experiment b) was repeated on prismatic specimen as shown in Figure 2 c). Finally, in order to avoid possible lateral leakage, water was supplied from below from a saturated sponge Figure $2 \mathrm{~d}$ ). Again in these prismatic specimens, after an hour of water supply, cracking is clearly visible in correspondence with previous experiments. Due to one smaller dimension, the rate of cracking also appears higher. Experiment d) was therefore selected to be performed under x-ray tomography and neutron radiography.

For the selected imbibition experiment, a fresh and presumably saturated cylindrical core - 


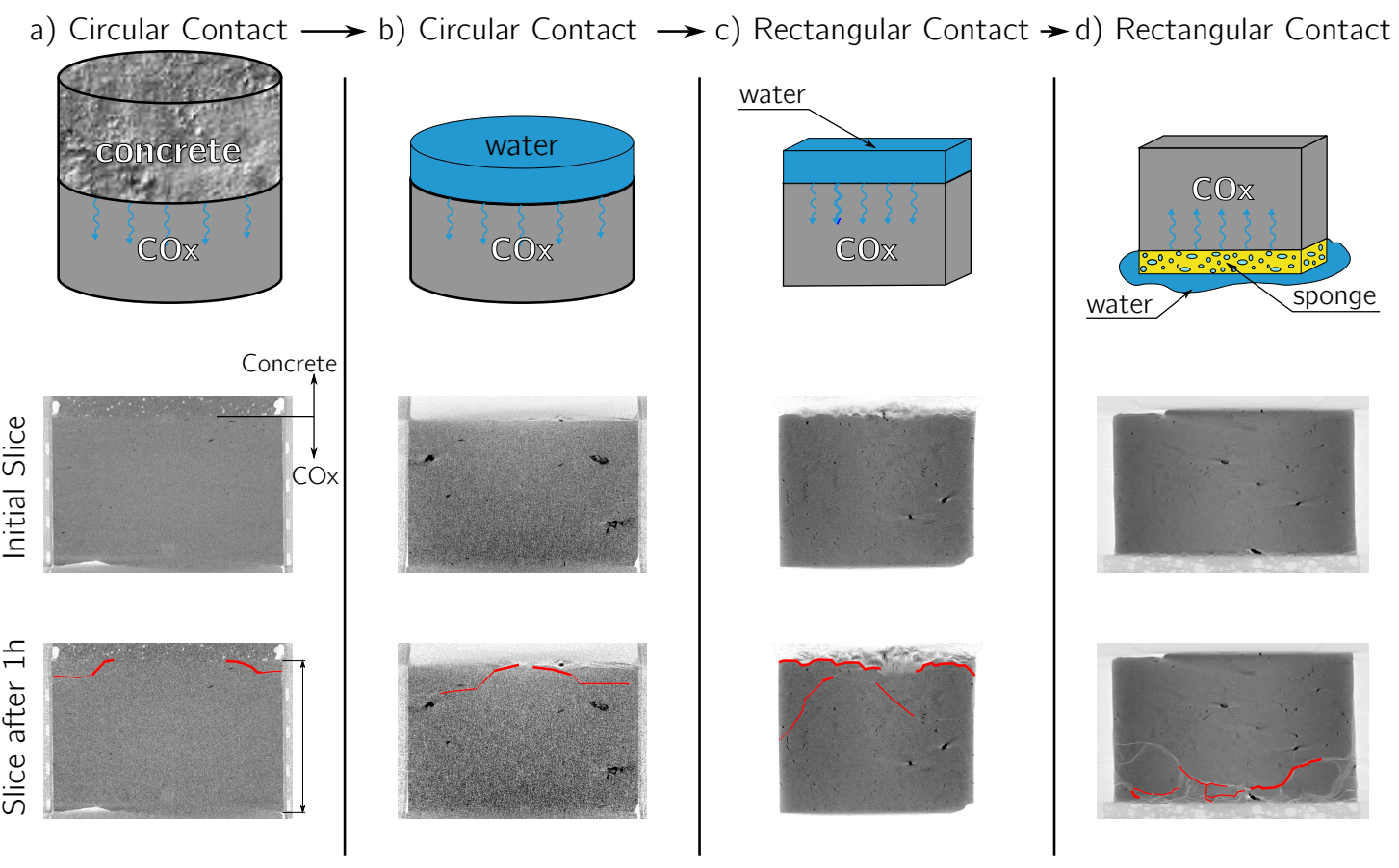

Figure 2: Illustration of the different steps of simplification which led to neutron scanning, with top: a sketch, middle: a vertical slice through an x-ray scan of an undeformed specimen, bottom: same specimen after an hour

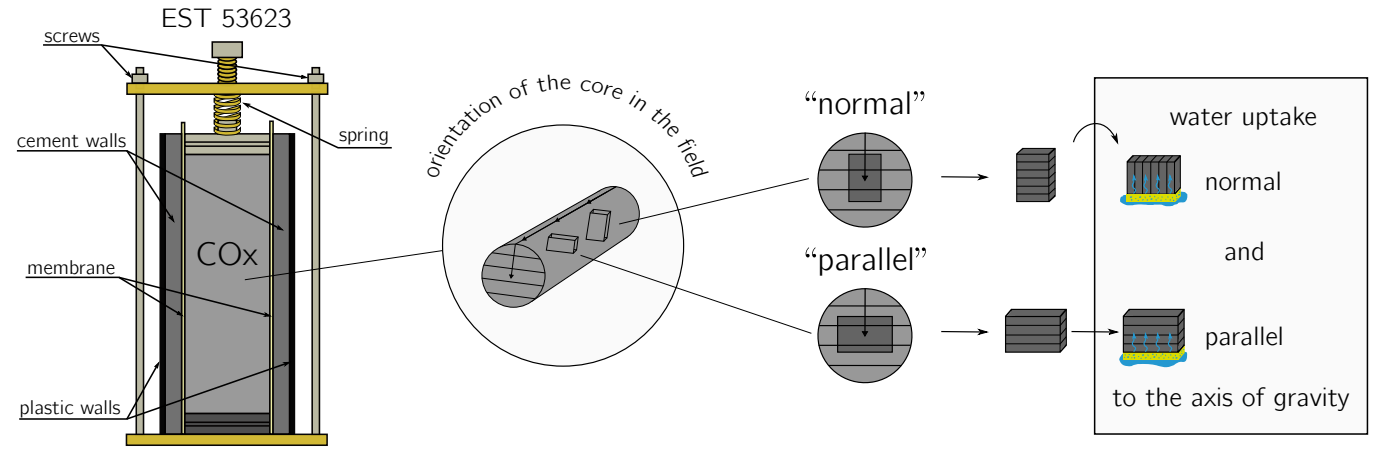

Figure 3: Description of the preparation of specimens from the COx core EST 53623 from Andra. From left to right: sealed COx core, laterally with a cement and a plastic wall and vertically pressurised with a compressed spring; extracted COx core; orientation of extraction of the two types of specimens, normal and parallel

EST 53623 - was opened. The long axis of the extracted core is normal to the axis of gravity. Four prismatic specimens, two normal and two parallel to the reported in-situ horizontal plane, were immediately cut with a diamond wire saw and rectified on all six faces with a belt sander (see Figure 3). Massive offcuts were used to measure the initial water content, giving an initial water content of $6.8 \%$ which is within the statistical range of water contents for saturation for this material, as reported in [19].

Four practically identical imbibition tests were performed independently under either neutron radiography or x-ray tomography - although in the future both imaging modalities will be available on the NeXT instrument. Samples destined for neutron radiography were scanned with x-ray tomography before imbibition.

For x-ray tomography, the scanner in Laboratoire 3SR is used. The scanner has a micro-focus source with Tungsten target, the acceleration of electrons into the target is set at $100 \mathrm{kV}$, and the resulting beam is not filtered. An amorphous silicon detector with CsI scintillator is used 


\begin{tabular}{|c|c|c|c|}
\hline Specimen Name & Dimensions $(\mathbf{m m})$ & Mass $(\mathrm{g})$ & $\begin{array}{c}\text { Time to experiment } \\
\text { after cutting }(\mathbf{h})\end{array}$ \\
\hline Parallel-X & $40.98 \times 32.60 \times 14.50$ & 45.29 & 3 \\
\hline Parallel-N & $44.35 \times 32.02 \times 14.51$ & 48.24 & $8 \frac{1}{2}$ \\
\hline Normal-X & $34.85 \times 31.92 \times 15.04$ & 38.88 & $5 \frac{1}{2}$ \\
\hline Normal-N & $36.20 \times 32.03 \times 14.13$ & 38.65 & $6 \frac{1}{2}$ \\
\hline
\end{tabular}

Table 1: Table describing the specimens tested

in 2x2 binning and given the non-cylindrical shape of the specimen 1200 projections (more than strictly necessary) are taken. The geometrical pixel size is $70 \mu \mathrm{m} / \mathrm{px}$ and the total scan time for one tomography is $5 \mathrm{~min}$. Reconstructions are performed with the XAct software provided by RX-Solutions (Annecy, France), with appropriate beam-hardening corrections applied.

For neutron radiography the NeXT instrument on the D50 beamline at the Institut LaueLangevin in Grenoble is used. The neutron beam has wavelengths $[1,10] \AA$ with a peak at $3 \AA$, making this a cold-neutron beam. For geometrical sharpness a $15 \mathrm{~mm}$ pinhole is used at around $10 \mathrm{~m}$ distance to the detector (giving a practically parallel beam) and a $5 \mathrm{~mm}$ thick graphite diffuser is used to homogenise the beam. A $50 \mu \mathrm{m}$ thick LiF scintillator is used and a $2048 \times 2048 \mathrm{~S}-\mathrm{CMoS}$ camera is used in $2 \times 2$ binning giving a pixel size of around $55 \mu \mathrm{m} / \mathrm{px}$. Ten second exposure is chosen used for each radiography as a compromise between image contrast and process speed. In all that follows radiographies are corrected by $I_{0}$, by the darkfield of the camera, gamma-ray streaks (which leave high-valued pixels in some radiographs) are removed by searching for high outliers, and radiographs are averaged with a median filter in groups of six, giving a total exposure time of $1 \mathrm{~min}$ per corrected radiography.

Regardless of the modality, each COx sample was held from the top with double-sided sticky tape. The bottom of the sample rests on a commercial kitchen sponge, cut to shape for the experiment, which was saturated rapidly with room-temperature tap water.

Neutron radiography

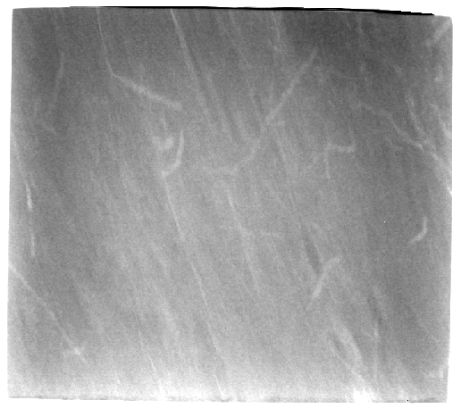

X-ray radiography*

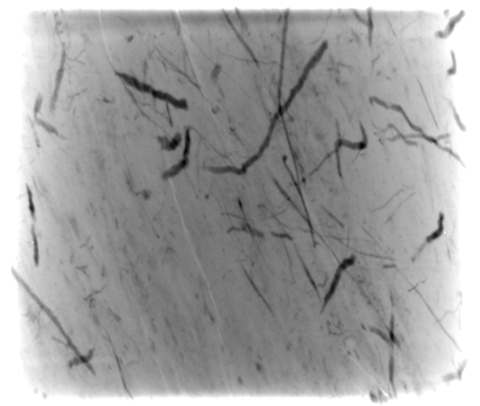

Neutron or x-ray attenuation

Low High

Figure 4: Neutron and x-ray radiography of sample Normal-N through the thinnest direction with holders masked from the image before water imbibition.

* Since the $\mathrm{x}$-ray data is collected with a divergent beam which gives different magnification through the depth of the specimen, the reconstructed 3D volume is virtually projected in parallel geometry in the same direction as the neutron radiography to obtain easier-to-compare attenuation images

Previous use of both modalities on geomaterials, for example by [20] show the utter complementarity of these two imaging techniques for the investigation of sand is a state of partial water-saturation. Figure 4 shows a comparison of the attenuation of the neutron and x-ray beams used in this work of sample "Normal-N" before imbibition. The inclusions in the clay matrix clearly attenuate more x-rays than the matrix, whereas they attenuate fewer neutrons than the matrix. 


\section{Acquired data and processing}

Figure 5 presents a number of neutron radiographies for the "-N" samples and central vertical slices through the reconstructed x-ray tomography volumes for the "X" samples. It is important to note that averaging together of the neutron radiographies over 5 min offers significant denoising, but in intervals where there is significant water movement can blur the radiography.

\subsection{X-ray tomographies}

In the vertical slices presented in Figure 5, x-ray tomography is clearly able to follow the deformation of the solid COx, but is not sensitive to water. Furthermore, in both "parallel" and "normal" samples, some pre-existing cracks are visible.

In the $3 \mathrm{D}+\mathrm{t} \mathrm{x}$-ray tomography timeseries acquired for samples Normal-X and Parallel-X during imbibition, a mask for the bounding surface of the sample is created in each time step. The objective of this mask is to define the "external" volume of the sample by filling cracks and removing the sample holder. An initial mask is obtained by applying a greyscale threshold on the reconstructed 16bit images of $[15400,65535]$ - see Figure 6 for the meaning of the greyvalues with respect to the histogram of reconstructed greyvalues. Cracks are filled by four cycles of morphological dilation of the selected solid phase with a radius of 3 pixels and four cycles of erosion; the number of cycles is selected as half of the maximum crack opening in pixels plus 1 pixel margin. The measure of volume of the mask in every timestep gives the outside volume of the deforming sample.

The detection of large cracks i.e., larger than 2 pixels can simply be done with a threshold. However, subpixel crack detection is more sensitive to noise and the beam hardening effect which are present in the images. A simple program was developed in Python using Numpy and Scipy[21, 22] for the detection of subpixel cracks: for any pixel within the mask described above, if its value is less than 12000 is it considered a void. For values higher than this threshold, they are considered cracks if their value is 2200 units lower than the local mean, which is itself calculated as a moving linear window \pm 9 pixels (i.e., $1.33 \mathrm{~mm}$ ) centred on the pixel of interest, avoiding values lower than 12000 (cracks) and higher than 26000 (dense inclusions). Since the boundary of the specimen is detected as a crack, the first two pixels on the boundary of the identified crack map are finally cropped (by masking with a twice-eroded overall sample mask).

\subsection{Neutron radiographies}

Neutron radiographies clearly reveal the entrance of water into the COx matrix. The principle for the analysis of the water entering the $\mathrm{COx}$ is based on the reasoning that the attenuation coefficient $\mu$ of the $\mathrm{COx}$ in its initial state of saturation will not change if the sample swells at constant mass (for example if it expands without losing or gaining water) - the mass attenuation coefficient $\mu_{m}=\mu / \rho$ will however change to reflect a decrease in density. Thus in a radiography of a sample swelling in all directions at constant mass, the swelling in the direction of the beam does not change the overall attenuation of the beam (changes in size in the direction of the beam are undetectable since increases in volume - and thus the length for the beam to cross - are cancelled out by the reduction in density), whereas swelling in the imaging plane will reduce the mass attenuation coefficient allowing more of the beam to cross the sample. If the increase in projected area in the detected radiography of the sample is taken into account, it is easy to show that:

$$
\text { Area }_{\text {initial }} \times \mu_{\text {initial }}=\text { Area }_{\text {swelled }} \times \mu_{\text {swelled }}
$$

Thus, at constant mass, for any swelling or contracting:

$$
\text { Area } \times \mu=\text { const. }
$$

It is important to note that since the reference $I_{0}$ images are acquired in air, when air invades the specimen, the projected area to be used is everything within the boundary of the specimen.

We are obviously not in a case of swelling at constant mass, since water invades the specimen, thus any departure from the above reasoning is assumed to be due to the entry of water. To this end, throughout the neutron radiographies acquired during water imbibition, the projected area of the specimen is carefully selected in the images using a mask for each radiography. Starting from 


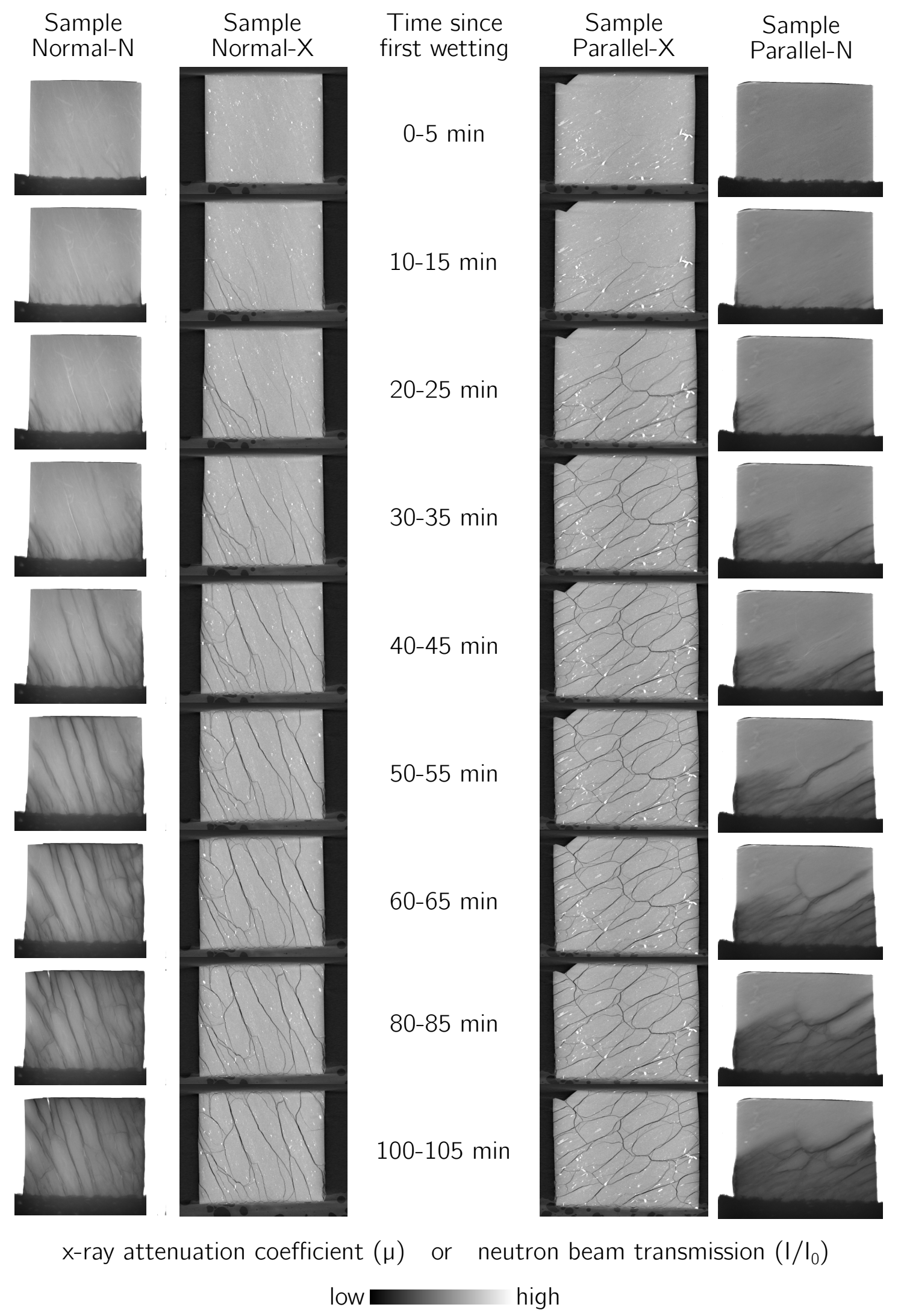

Figure 5: Selected in-situ images acquired during imbibition for the four samples in Table 1. X-ray data is presented as a vertical slice in the middle of the specimen, whereas neutron radiographies are averaged together in 5-minute intervals in order to match the x-ray tomography times. The structure holding the sample from the top has been manually erased from neutron radiographies. Accuracy on times is \pm 90 s 


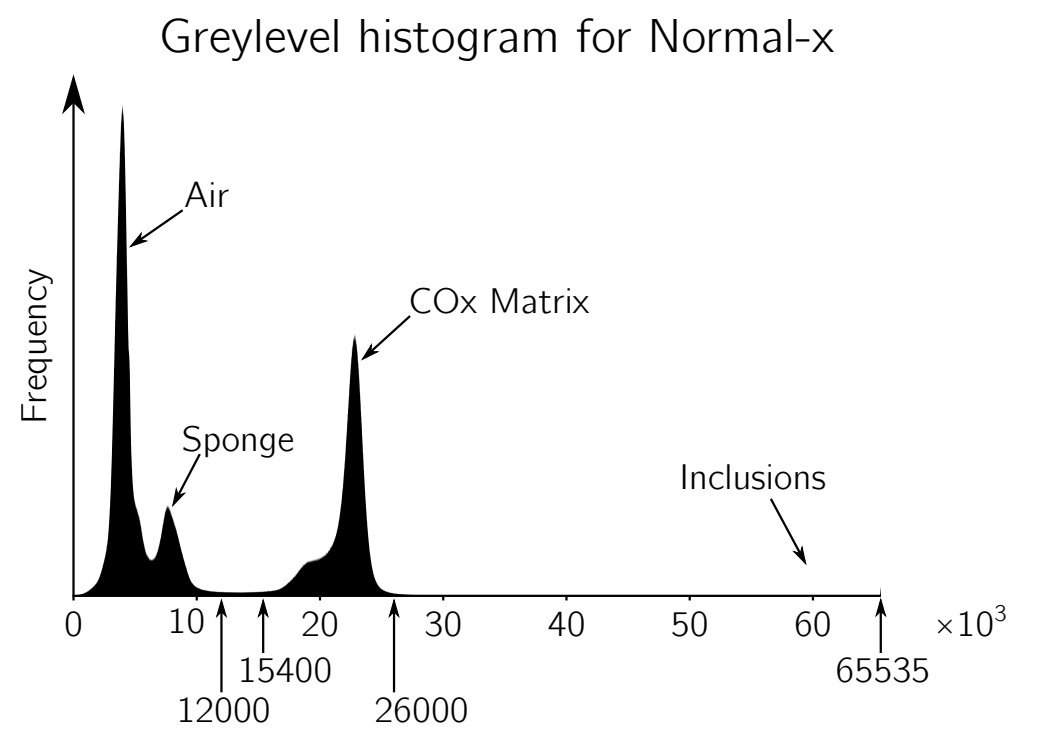

Reconstructed 16bit x-ray attenuation greylevel

Figure 6: Greylevel histogram of the reconstructed x-ray tomographies

the initial dry image, the expected average attenuation in each subsequent radiograph is calculated:

$$
\mu_{\text {current }}=\frac{\mu_{\text {initial }}}{\text { Area }_{\text {initial }}} \times \text { Area }_{\text {final }}
$$

This is compared to the measured mean attenuation value, and any difference is ascribed to the entrance of water into the specimen. The change in attenuation can thus be directly linked to a mean "thickness" of water entering the masked area using the fit in Figure 1. The mean thickness of water over the projected area of the specimen thus gives a relatively direct measurement of water volume entering the specimen with one minute resolution.

\section{Sample-scale measurements}

In this section, measurements at the scale of the sample are presented. These measurements use the analysis of the acquired x-ray tomography and neutron radiography data detailed in the previous section in an average sense.

Figure 7 shows the evolution of sample-wide quantities that can be obtained: x-ray tomography data is used to measure the change in volume of the external boundary of the specimen (using the mask) and the volume of visible cracks using the method described above, and neutron radiographies are used to measure water entering the specimen. The evolution of these three volumes is shown from the beginning of imbibition process in Figure 7 for both types of sample: Parallel (squares) and Normal (circles), normalising each by the initial volume of the sample as obtained from x-ray tomography. It is important to note that the sub-pixel sensitivity of the crack detection is expected to significantly overestimate crack volume for smaller-than-pixel crack openings. A small volume of cracks is detected even at the beginning of the test, which on close inspection of the x-ray tomography images makes sense, since some hairline cracks are present in the samples before imbibition, presumably due to the stress release with respect to site conditions.

The measurement of the change in water volume inside the boundary of the specimen is obtained from the analysis of the neutron radiographies. The change in attenuation of the specimen (taking into account its change of area/volume) is assumed to be entirely ascribable to water entering the specimen, and is converted to a volume using the fit to Beer-Lambert in Figure 1. The maximum increase of attenuation measured (396 min after imbibition for Parallel-N) corresponds to a path length of neutrons in water of $2.13 \mathrm{~mm}$ which is just within the area of confidence of the fit. This measurement cannot be verified directly due to experimental difficulties (the sample falls apart after $2 \mathrm{~h}$ imbibition and in neutron radiography the sample becomes radioactive and cannot be 


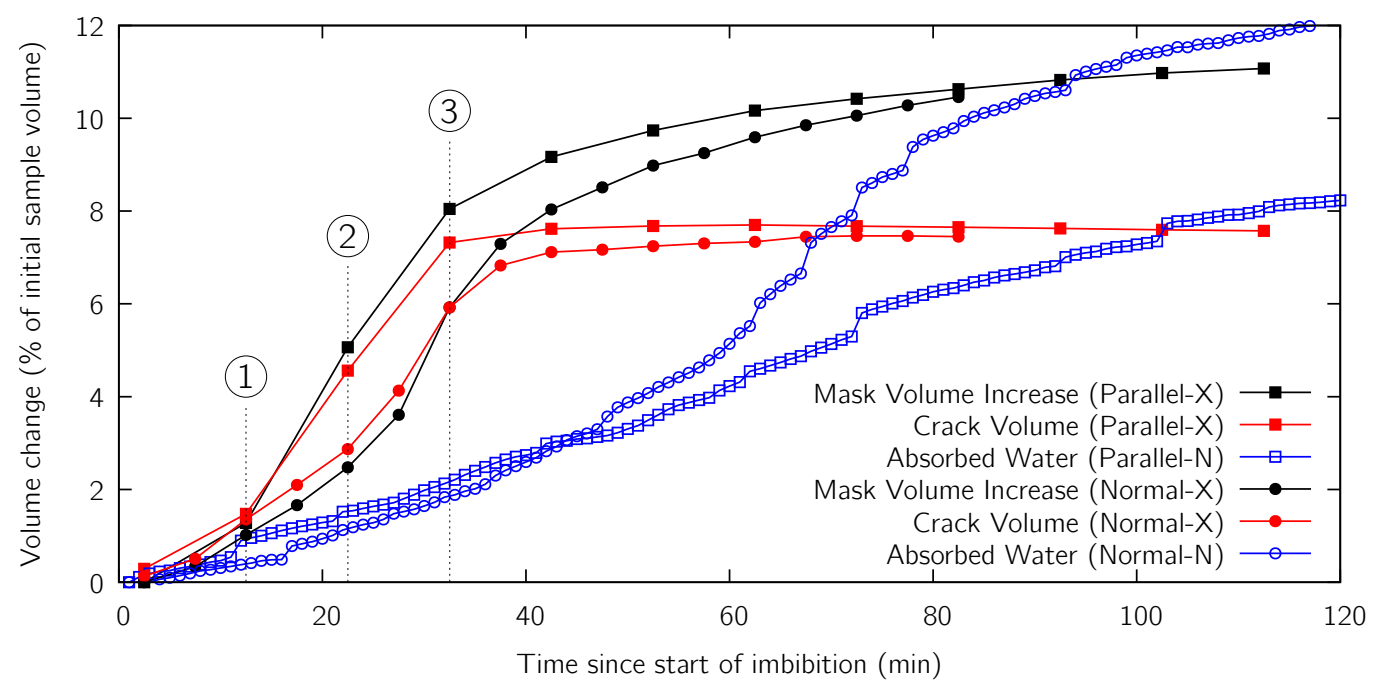

Figure 7: Macroscopic measurements of sample evolution from x-ray tomography (mask and crack volumes) and Neutron radiography (water volume). It is important to note that for the x-ray scan the time is taken as the middle of the 5-minute scan, and that Normal and Parallel samples are not identical. Accuracy on times is $\pm 90 \mathrm{~s}$

handled immediately) but the measured volumes correspond satisfactorily to the low-quality mass measurements after x-ray tomography tests.

The upshot of Figure 7 is that the imbibition process causes a rapid change of volume (for both directions of exposed surfaces) in the COx samples tested, with the peak rate between 10 and 40 minutes. In this early stage, it appears that (again for both tested orientations) most of the sample's increase in volume is due to large cracks that appear and develop. In both samples tested, after about 50 minutes crack volumes then become relatively constant in the observed time. The water, which causes the cracks, steadily enters the sample at a significantly lower rate than the crack propagation, however continues to do so after the cracks have stopped increasing in volume, thus the phase starting from $50 \mathrm{~min}$ onwards appears to be one of water uptake into the matrix without cracking at the scale observed.

\section{Local measurements}

\subsection{Local observations}

Full-field imaging also allows local processes within the sample to be followed. Figure 8, for example shows the relative success of the crack identification algorithm presented above in detecting small cracks in three selected x-ray tomography images, representing points of significant cracking activity (marked as 1,2,3 in Figure 7). For both types of samples, a central, vertical slice through the original greyscale volumes is presented, alongside the same slice through the detected crack field, as well as a $3 \mathrm{D}$ rendering of the entire detected crack field.

The success of the crack detection algorithm means that, for example, the structure of the cracks (size and number of connections) could be analysed with ease.

Another local measurement of key importance is the interplay between cracking and water distribution in the sample. The sample-scale comparisons presented in Figure 7 seem to indicate a good correspondence between x-ray and neutron measurements, however since (slightly) different samples are followed with x-rays and neutrons, a direct local comparison cannot be made. The cracking that can clearly be seen in x-rays can also be seen with neutron radiography (although in this case its volume cannot be measured directly).

Figure 9 presents neutron radiographies for sample Normal-N with a higher time resolution (and therefore more noise) than Figure 5 with two zooms into areas of the sample: the larger area (top line) presents minute-averaged radiographies showing the development of three cracks (A, B and C) in the middle of the sample, whereas the smaller area (bottom line) shows ten- 


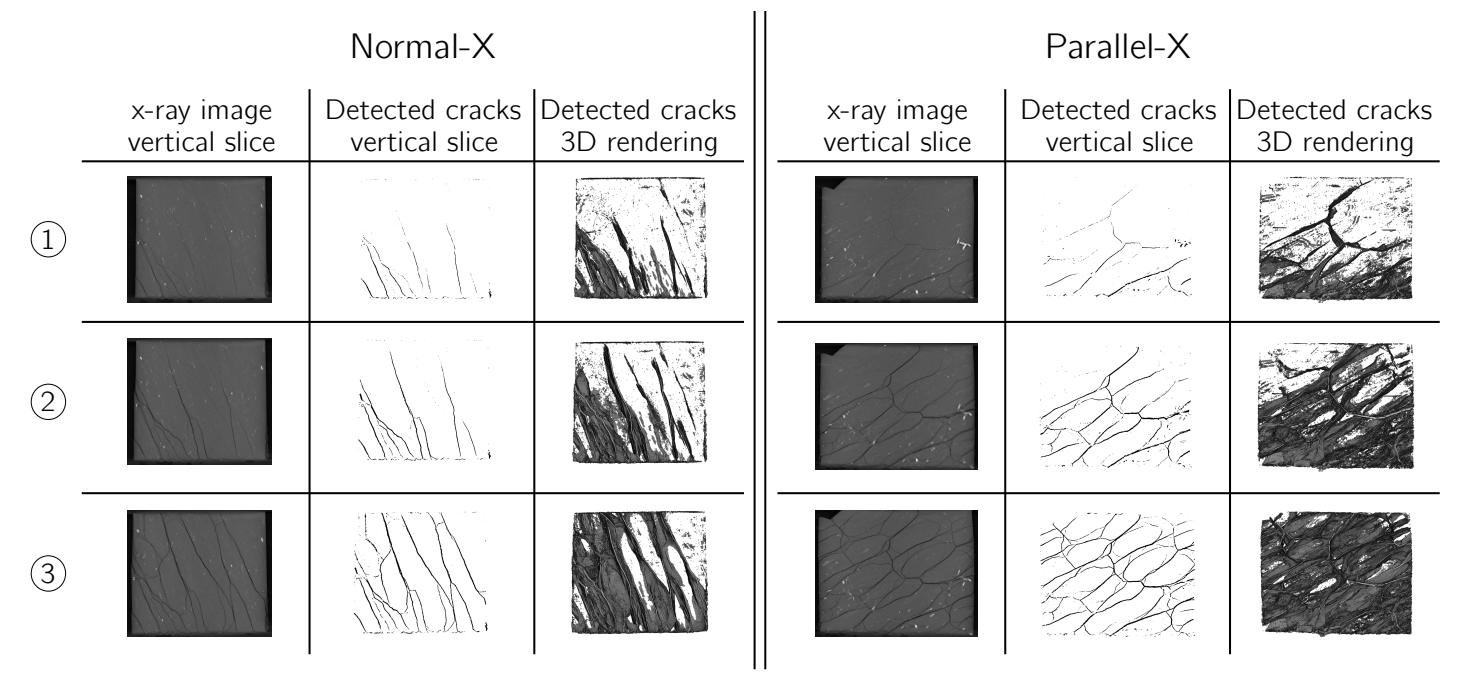

Figure 8: Crack state for the two types of samples, during the three time-steps indicated in Figure 7 , when the the crack propagation is rapid

second radiographies of crack C. The top line of Figure 9 shows some interesting features of crack development with the opening of a dry crack, which is then filled with water from both sides (clearly indicating a structural effect of connected water in the specimen) for crack A, followed by $\mathrm{B}$ and $\mathrm{C}$. The bottom line of Figure 9 aims to present the kinematics of crack opening, followed by what must be capillary rise. This relatively simple conclusion would have been very difficult to be certain about with x-ray tomography due to the low contrast that water presents with respect to air.

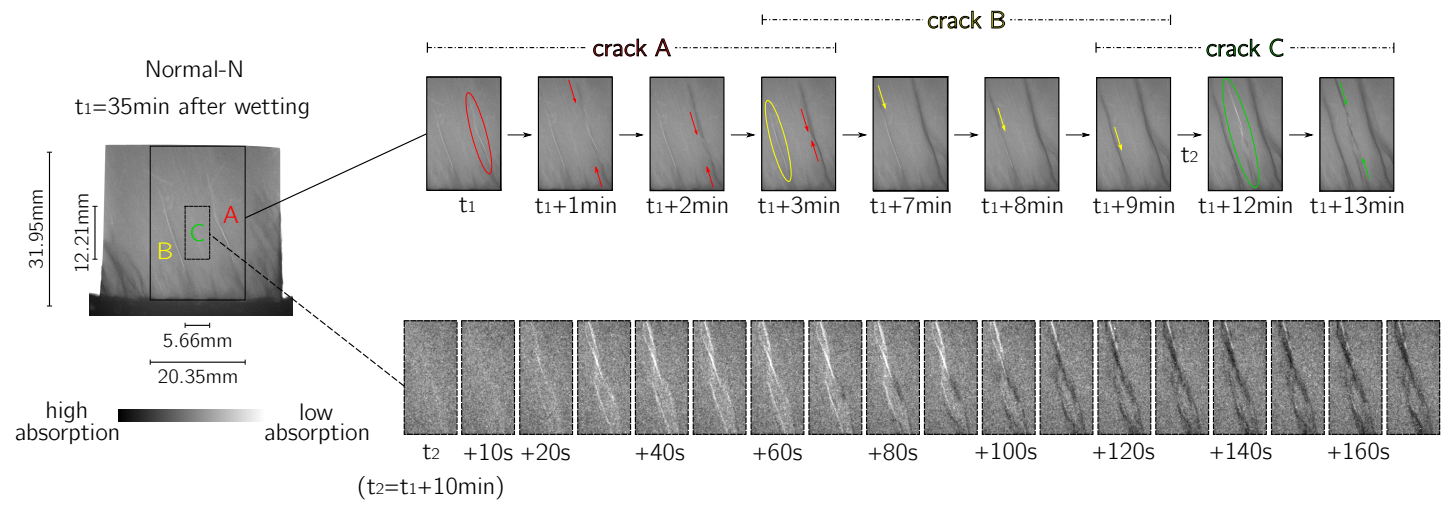

Figure 9: Neutron imaging observation of the opening of three different cracks $(A, B, C)$ in time and demonstration of the sequence of the phenomena: mechanical cracking followed by water penetration for the Normal-N sample. Upper row: Sequence of the three different cracks and water penetration in time. Lower row: Sequence of crack $\mathrm{C}$ opening and water penetration in time

Figure 10 also presents neutron radiographies of cracks opening followed by water invasion, however for the sake of an illustrative example, an inset central vertical slice from x-ray tomography is presented where a clear air-water front can be seen within an open crack. Given the size and the contrast of the x-ray inset, it is once more obvious that neutrons allow a much clearer observation of the water front. 


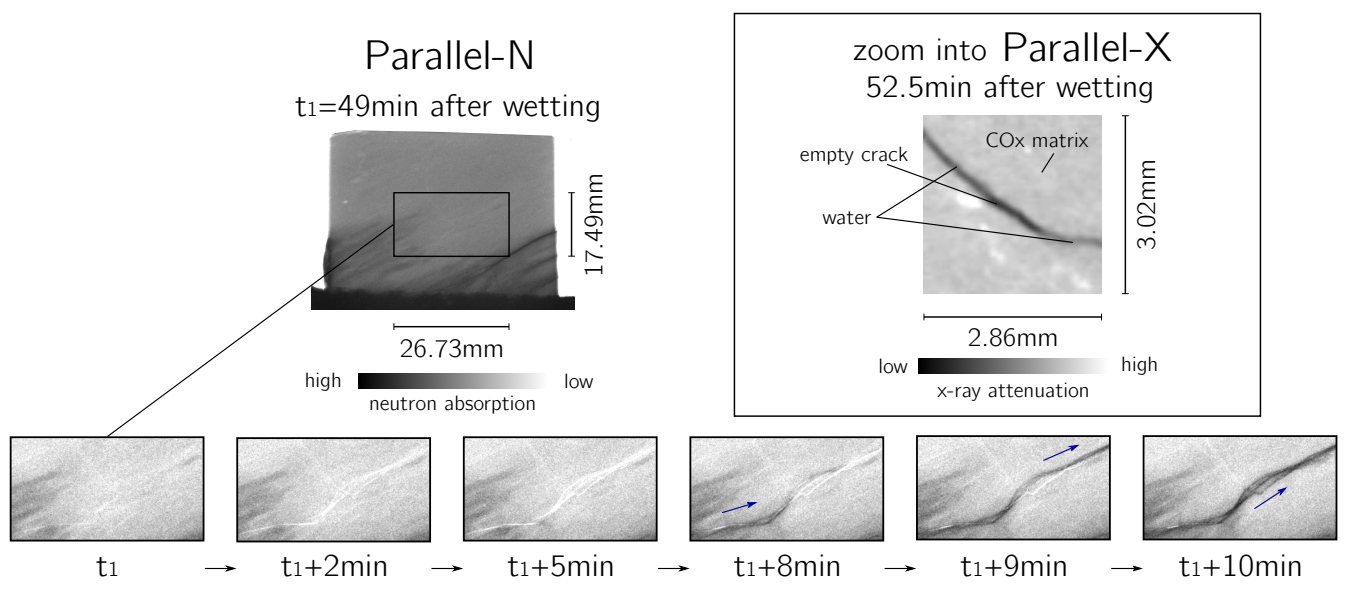

Figure 10: Neutron imaging observation of crack opening and water penetration for the Parallel-N sample. Inset vertical slice from Parralel-X sample

\subsection{Digital Volume Correlation}

The time-resolved x-ray tomographies, of which vertical slices are shown in Figure 5, are ideally suited to following the solid material's evolution as the specimen deforms. The identification of cracks and the specimen's external volume have been presented above, however a finer measurement of material evolution using Digital Volume Correlation (DVC) is also possible to the fine texture present in the images. Here we use a new development library called RANDOMWAFFLE. In this case, a regular mesh of nodes spaced 18 pixels (i.e., $70 \mu \mathrm{m} \times 18=1.26 \mathrm{~mm}$ ) apart defines measurement nodes. For each node, a representative sub-volume centred on its 3D position is extracted from the $3 \mathrm{D}$ volume which represents the reference configuration. The DVC code then attempts to match this reference sub-volume in the next 3D volume. Subpixel accuracy is obtained with 3rd order image interpolation, and a transformation operator is obtained, mapping each 3D point into the next image.

Given the significant disturbance created by the cracks, DVC is performed incrementally between pairs of images. Strains are calculated using TomoWARP2[23] using a large strain hypothesis, and using only the displacements from DVC. The increments of the trace of the locallycalculated strain tensor are presented in Figures 11 and 12, and referred to as volumetric strain. It is important to note that during cracking, the transformation operator is obviously unable to capture this behaviour, causing large apparent strains in the cracks.

Figure 11 illustrates vertical slices through the field of incremental volumetric strain for the "parallel" sample. The disturbance due to cracking is evident, presenting saturated values of strain, which in this experiment is most striking between the second and third increments. However, before and after cracking, swelling is clearly measurable within the sample.

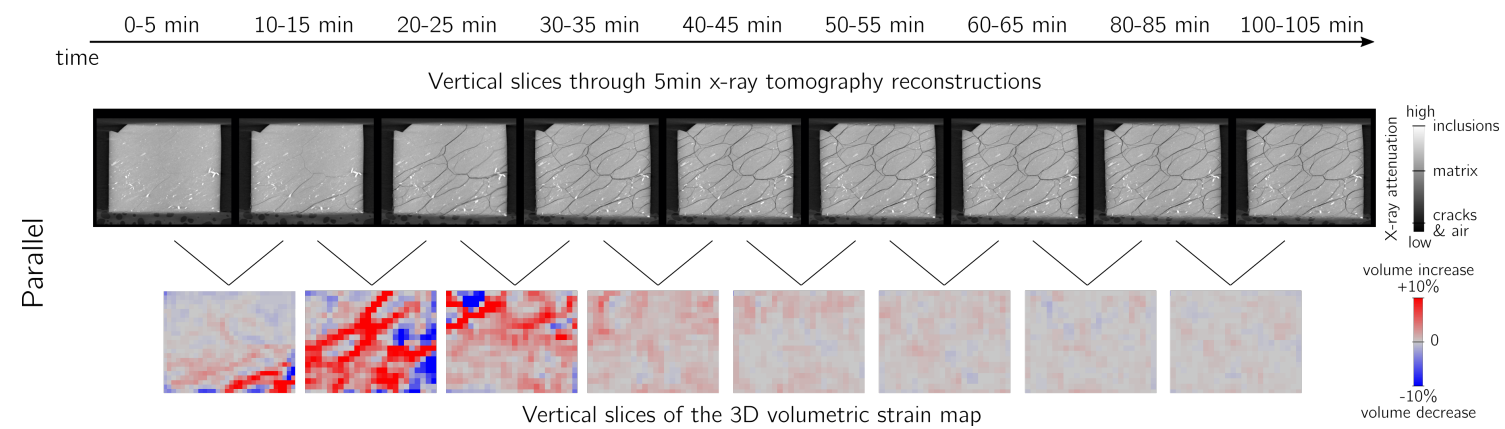

Figure 11: Series of vertical slices with time (parallel orientation). Top: x-ray tomography reconstructed images, Bottom: incremental volumetric strain maps (from incremental DVC) 
Figure 12 also shows x-ray images and incremental volumetric strain maps, and adds the corresponding neutron radiography images. It is important to note that the samples are similar but not identical. This being said there is a good correspondence between neutron and x-ray datasets for this test. Figure 12 presents an important perspective for further exploration of the direct measurement of hydro-mechanical coupling in this material: the combination of techniques presented allows a change of solid volume to be related to water entry. When this "multimodal" scanning is available simultaneously for the same specimen, this will allow the important measurement of the local change in water content, by updating the local value of solid density from the volumetric strain.

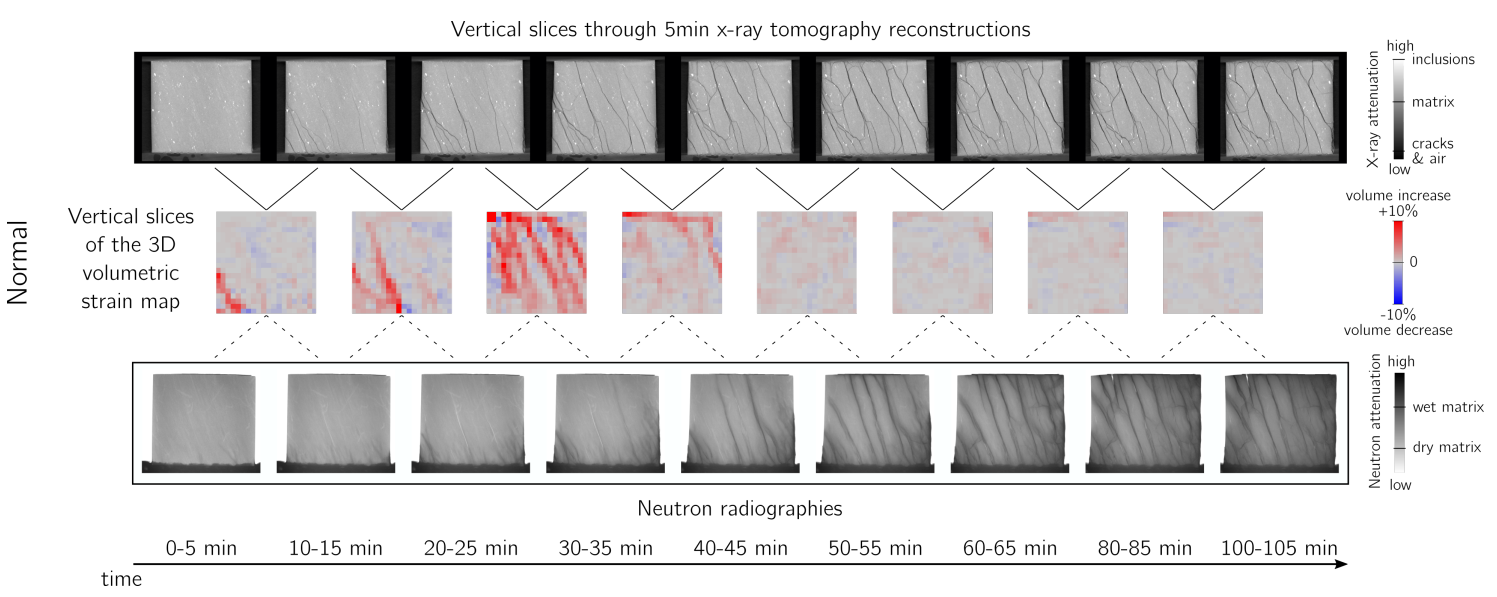

Figure 12: Series of vertical slices with time (parallel orientation). Top: x-ray tomography reconstructed images, Middle: incremental volumetric strain maps (from incremental DVC), Bottom: Neutron radiographies of similar sample

\section{Discussion}

The observed processes can be explained purely as a hydro-mechanical interaction. The samples studied - as well as those prepared for testing in the SInC box - are almost certainly in a state of partial saturation due to both water losses (evaporation during sample preparation in the lab, and possibly during transport) as well as an increase of volume of the soil skeleton due to elastic rebound due to unloading from the site stress condition. As reported in [24], the suction stresses generated in COx even for small changes of water content are significant, for example $150 \mathrm{MPa}$ of suction is required to dry their samples from $6 \%$ water content to $2.5 \%$. The suction necessarily present in the specimens studied here applies an internal effective stress, which holds the sample together despite the presence of cracks (from the tunnel's EDZ and/or induced by coring and sample preparation). Thus, when an unconfined specimen is placed in rapid contact with an abundance of free water, natural capillary rise leads to a local breakdown of suction. The breakdown of suction locally decreases the effective stress and leads to swelling. This differential swelling allows existing cracks to open, and potentially new ones to form, with an preferred orientation depending on the clay-rock's orientation in the bedding plane.

As is clearly shown throughout the measurements presented in this work, a large degree of cracking occurs when the COx samples are put into contact with water. Figures 9 and 10 are strong evidence for the propagation of cracks in the samples before the arrival of water around the crack. This is consistent with the hypothesis that suction is keeping the sample together, and as soon as it is removed by the presence of free water, effective stress falls dramatically and the sample disintegrates.

The macroscopic volume evolutions presented in Figure 7 show, for both sets of samples studied with in-situ x-ray tomography, a rapid increase of the external volume initially due to cracks that are detectable at the resolution used. The crack volume detectable at this scale stabilises between 7 and $8 \%$ for both sample orientations, whereas the external volume continues to increase due to swelling or cracks below the resolution. The water volume arriving into similar samples studied 
with neutron radiography appears to exceed the total volume increase of the sample, which is perfectly consistent with a state of partial saturation in the initial sample. This final conclusion however is weak, due to different samples being studied.

\section{Conclusions and perspectives}

This paper presents some of the sample-scale and local-scale measurements that can be obtained on the Callovo Oxfordian clay-rock with the highly complementary contrast mechanisms of x-ray and neutron beams. The uptake of liquid water in unconfined $\mathrm{COx}$ specimens has been studied, allowing a direct measurement of the rate of sample volume increase (both globally and locally), crack growth and water uptake. Even though the unconfined testing conditions are not representative of the in-situ under stress state, the cracking response of the clay-rock appears to be governed by hydro-mechanical interactions. The cracking observed in the preparation of samples to simulate the clay-rock/concrete interface, is therefore interpreted as a breakdown of suction (and therefore effective stress) due to water penetration.

The principal crack orientation of the "normal" samples appears to be perpendicular to the cracks of the "parallel" samples, revealing the anisotropy of the Callovo Oxfordian clay-rock. A favourable cracking orientation could be suspected for the "parallel" samples, more results are however necessary for a clearer understanding.

The measurements resulting from the combination of the two imaging techniques reveal a rapid crack opening and propagation in the COx samples within 30-40 minutes of contact with free water, which then appears to stabilise. The water penetrates the samples significantly more slowly, but steadily, reaching the samples' increased volume (filling open cracks) after around $120 \mathrm{~min}$.

With the truly combined simultaneous neutron and x-ray tomography setup expected to come online on the NeXT instrument on the D50 beamline at the ILL in early 2018, a full coupling between measurements of the same sample can be done, allowing for example, the comparison of water uptake (neutrons) to volumetric strain obtained with DVC on the x-ray tomography volumes.

A clear path for this combination of tools is to look at this material (ideal for neutrons since its hydraulic behaviour is key, all while having low water content in-situ) under stresses of interest for the site.

\section{Acknowledgements}

Simon Salager and Pascal Charrier in Laboratoire 3SR are gratefully acknowledged for the simple but brilliant idea of using a sponge as a water reservoir. We would like to thank all the people who have helped make NeXT a reality, especially Benjamin Giroud and Jérôme Beaucour. Laboratoire 3SR is part of the LabEx Tec 21 (Investissements d'Avenir grant agreement $n^{\circ}$ ANR-11-LABX0030). The first author would like to thank Andra for the financial support and the samples that allowed these experiments to happen.

\section{References}

[1] Armand, G., Noiret, A., Zghondi, J., \& Seyedi, D. M. (2013). Short-and long-term behaviors of drifts in the Callovo-Oxfordian claystone at the Meuse/Haute-Marne Underground Research Laboratory. Journal of Rock Mechanics and Geotechnical Engineering, 5(3), 221-230.

[2] Menaceur, H., Delage, P., Tang, A. M., \& Conil, N. (2015). The thermo-mechanical behaviour of the Callovo-Oxfordian claystone. International Journal of Rock Mechanics and Mining Sciences, 78, 290-303.

[3] Zhang, C., \& Rothfuchs, T. (2004). Experimental study of the hydro-mechanical behaviour of the Callovo-Oxfordian argillite. Applied Clay Science, 26(1), 325-336.

[4] Vinsot, A., Mettler, S., \& Wechner, S. (2008). In situ characterization of the Callovo-Oxfordian pore water composition. Physics and Chemistry of the Earth, Parts A/B/C, 33, S75-S86. 
[5] Armand G., Bumbieler F., Conil N., de la Vaissière R., Bosgiraud J.-M., Vu M.N., 2017, Main outcomes from in situ thermo-hydro-mechanical experiments programme to demonstrate feasibility of radioactive high-level waste disposal in the Callovo-Oxfordian claystone, Journal of Rock Mechanics and Geotechnical Engineering, Volume 9, Issue 3, June 2017, p. 415-427

[6] de La Vaissière R., Armand G., Talandier J., 2015, Gas and water flow in an excavation-induced fracture network around an underground drift: A case study for a radioactive waste repository in clay rock, Journal of Hydrology 521, pp141-156

[7] Stavropoulou, E., Briffaut, M., Dufour, F., Camps, G., \& Boulon, M. (2017). "A new apparatus for testing the delayed mechanical behaviour of interfaces: The Shearing Interfaces Creep box (SInC box)". Comptes Rendus Mécanique.

[8] Pham Q.T., Vales F., Malinsky L., Nguyen Minh D., Gharbi H., 2007, Effects of desaturationresaturation on mudstone. Phys Chem Earth 32:646-655

[9] Bornert M, Vales F., Gharbi H., Nguyen Minh D., 2010, Multiscale full-field strain measurements for micromechanical investigations of the hydromechanical behavior of clayey rocks. Strain 46:33-46

[10] Guillon T., Giot R., Giraud A., Armand G., 2012, Response of Callovo-Oxfordian claystone during drying tests: unsaturated hydromechanical behavior Acta Geotechnica 7:313-332

[11] Matray J.-M., Savoye S., Cabrera J., 2007, Desaturation and structure relationships around drifts excavated in the well-compacted Tournemire's COx claystone (Aveyron, France), Eng Geol ,90:1-16.

[12] Hedan S., Fauchille A.-L.,a, Valle V., Cabrera J, Cosenza P., 2014, One-year monitoring of desiccation cracks in Tournemire COx claystone using digital image correlation International Journal of RockMechanics \& MiningSciences 68(2014)22-35

[13] Armand G., Djizanne H., Zghondi J, de La Vaissière R., Talandier J., Conil N., 2016, Inputs from in situ experiments to the understanding of the unsaturated behaviour of CallovoOxfordian claystone, E-UNSAT 2016, DOI: 10.1051/20160903004

[14] Montes H.G., Duplay J., Martinez L., Escoffier S., Rousset D., 2004. Structural modifications of Callovo-Oxfordian COx claystone under hydration/dehydration conditions, Appl Clay Sci 25:187-194

[15] Wang L., Bornert M., and Chanchole S., 2013. Micro-Scale Experimental Investigation of Deformation and Damage of Argillaceous Rocks under Hydric and Mechanical Loads. Poromechanics V, 1635-1643

[16] A. Tengattini, D. Atkins, B. Giroud, E. Andò, J. Beaucour, G. Viggiani (2017). "NeXTGrenoble, a novel facility for Neutron and X-ray Tomography in Grenoble". Proceedings ICTMS2017

[17] Lenoir, N., Bornert, M., Desrues, J., Bésuelle, P., \& Viggiani, G. (2007). "Volumetric digital image correlation applied to X-ray microtomography images from triaxial compression tests on argillaceous rock". Strain, 43(3), 193-205.

[18] Rinard, P. (1991). "Neutron interactions with matter". Passive Nondestructive Assay of Nuclear Materials, 357-377. https://fas.org/sgp/othergov/doe/lanl/lib-www/la-pubs/ 00326407.pdf

[19] Armand, G., Leveau, F., Nussbaum, C., de La Vaissiere, R., Noiret, A., Jaeggi, D., ... \& Righini, C. (2014). "Geometry and properties of the excavation-induced fractures at the Meuse/Haute-Marne URL drifts". Rock Mechanics and Rock Engineering, 47(1), 21-41.

[20] Kim, F. H., Penumadu, D., Gregor, J., Kardjilov, N., \& Manke, I. (2012). "High-resolution neutron and X-ray imaging of granular materials". Journal of Geotechnical and Geoenvironmental Engineering, 139(5), 715-723. 
[21] Stéfan van der Walt, S. Chris Colbert and Gaël Varoquaux. "The NumPy Array: A Structure for Efficient Numerical Computation", Computing in Science \& Engineering, 13, $22-30$ (2011), DOI:10.1109/MCSE.2011.37 (publisher link)

[22] Jones E, Oliphant E, Peterson P, et al. 11SciPy: Open Source Scientific Tools for Python, 2001-", http://www.scipy.org/ [Online; accessed 2017-05-07]

492

[23] E. Tudisco, E Andò, R. Cailletaud, S.A. Hall, (2017). "TomoWarp2: a local Digital Volume

493 Correlation code", SoftwareX, Accpeted for publication

494

[24] Menaceur, H., Delage, P., Tang, A. M., \& Talandier, J. (2016). "The status of water in swelling shales: an insight from the water retention properties of the Callovo-Oxfordian claystone". Rock Mechanics and Rock Engineering, 49(12), 4571-4586. 